\title{
A Mean Curvature Regularized Based Model for Demodulating Phase Maps from Fringe Patterns
}

\author{
Carlos Brito-Loeza, Ricardo Legarda-Sáenz, Arturo Espinosa-Romero \\ and Anabel Martin-Gonzalez* \\ CLIR at Facultad de Matemáticas, Universidad Autónoma de Yucatán, México.
}

Received 12 May 2017; Accepted (in revised version) 11 November 2017

\begin{abstract}
We introduce a variational method for demodulating phase maps from fringe patterns. This new method is based on the mean curvature of the level sets of the phase surface that is used for regularization. The performance of the method is illustrated with both synthetic and real data.
\end{abstract}

AMS subject classifications: 35Q60, 35A15, 65K10, 78M30

Key words: Fringe analysis, interferometry, high-order regularization, variational models.

\section{Introduction}

Fringe analysis techniques are very popular to estimate with reasonable accuracy physical quantities such as shape of objects, deformation, refractive index and temperature fields. They achieve these goals by recovering the local phase from one or a collection of interference fringe pattern images. The mathematical model of a fringe pattern is described by the equation

$$
u=a+b \cos (\psi+\phi),
$$

where $a$ is the background illumination, $b$ is the amplitude modulation, $\psi$ is the spatial carrier frequency and $\phi$ is the phase map to be recovered. The problem of recovering not only $\phi$ but also $a$ and $b$ from the above equation is an ill-posed problem. Having only Eq. (1.1) to recover the three unknowns $a, b$ and $\phi$, plus the nonlinearity of the cosine function makes the problem very challenging. Recently, variational techniques that aim to reduce uncertainty of the solution by introducing more information into the model

*Corresponding author. Email addresses: carlos .brito@correo.uady.mx (C. Brito-Loeza), rlegarda@correo.uady.mx (R. Legarda-Sáenz), eromero@correo.uady.mx (A. Espinosa-Romero), amarting@correo.uady.mx (A. Martin-Gonzalez) 
by means of regularization of the unknown variables have proved to deliver a feasible solution to this problem, see $[10,17,30]$ and references therein. The new information introduced in the form of a regularizer defines the properties of the variational solution so a careful selection is advised.

The paper is organized as follows: in Section 2 we review the TV model, its virtues and drawbacks. In Section 3 we introduce a new curvature based model. In Section 4 we present the numerical solution of the Euler-Lagrange equations. In Section 5 are the experimental results and Section 6 is used to present our conclusions. In the Appendix at the end we present the derivation of the Euler-Lagrange equations.

\section{Review}

The use of regularization to process phase maps can be traced back to the works of [9, $13,18,25$ ] and references therein. In those works some sort of smooth regularization was used with the purpose of stabilizing the solution of the proposed algorithms. However, it is not until very recently that cutting-edge variational techniques, already successfully proved in the field of image processing, have started to being applied to the modeling of interferometry problems. For instance, the very popular Total Variation (TV) regularizer was used in [19] for measurement of planar refractive index profiles with rapid variations in glass using interferometry. Likewise, in [22] the authors proposed a TV based singleshot interferogram analysis for accurate reconstruction of step phase objects.

A work of particular interest to us is the one we presented in [17], where a variational method was proposed for recovering a discontinuous phase map from a single pattern. This method, applied TV regularization to all three unknowns $\phi, a$ and $b$ as it is shown below

$$
\begin{aligned}
\underset{a, b, \phi}{\operatorname{argmin}} T V(a, b, \phi, g) \equiv & \left\{\int_{\Omega}(u-g)^{2} d \Omega+\lambda_{1} \int_{\Omega}|\nabla a| d \Omega\right. \\
& \left.+\lambda_{2} \int_{\Omega}|\nabla b| d \Omega+\lambda_{3} \int_{\Omega}|\nabla \phi| d \Omega\right\},
\end{aligned}
$$

where $\Omega \subseteq \mathcal{R}^{2}$ is the domain of integration, $g$ is a given fringe pattern and $\lambda_{i}>0, i=1,2,3$ are regularization parameters.

The distinctive feature of (2.1) is that it allows the recovering of sharp phase transitions, something that other methods, such as those based on $L_{2}$ regularization, fail to deliver. However, recent studies $[4,24,29]$ have shown that TV regularization brings some unwanted inconveniences as well. The most known being staircasing and the loss of signal height.

The contribution of this paper is to present a different regularizer, based on the mean curvature of the level sets of $\phi$, which overcomes these drawbacks while still retaining the nice properties of the TV model. 


\section{Staircasing}

This phenomenon, which appears in TV regularized image denoising models, such as [20], causes images to look blocky and has been extensively studied $[5,6,16]$. Staircasing can easily been observed in regions with low curvatures values and maybe explained as the preservation of monotonicity of neighboring values. The model in (2.1) will therefore transform smooth or piecewise smooth signals into piecewise constant signals.

\section{Loss of signal height}

When working on phase retrieval, the right selection of the phase regularizer is quite relevant since it imposes a set of constraints defining the properties of the phase map recovered and therefore a careful selection should be done. In metrology, the height of $\phi$ may represent the amount of deformation that a given physical structure has suffered due the action of forces acting on it, hence an accurate recovery is expected from the variational solution. On this matter, it has been documented that the TV regularizer does not preserve the height of processed signals. In variational image denoising, the authors in [24] managed to derive a rule for piecewise constant signals which states that "for piecewise constant image features the change in image intensity due to TV regularization is directly proportional to the regularization parameter and inversely proportional to the scale of the image feature". For the model (2.1), the loss of signal height for $\phi$ is given by

$$
\delta=\frac{\lambda_{3}}{\text { scale }}
$$

where $\delta$ stands for the change in signal height, $\lambda_{3}$ the regularization parameter and scale is defined as the ratio of the area of the feature to its boundary length.

On one hand, this inconvenient property of the TV regularizer makes it not suitable for metrology applications where an accurate recovery of the phase map is critical. On the other hand, we argue that TV, or even $L_{2}$ regularization, might be the right choice for $a$ and $b$ since in most applications, they are found to be piecewise constant or almost constant functions.

The analysis above shows that $\phi$ would benefit from a better selection of the regularizer. Recently, strong evidence showing that curvature based regularizers such as mean curvature [29] and Gaussian curvature [4] have much better properties than those of TV has been presented. Curvature regularizers, as proved in $[4,29]$ do not damage the height of the phase while still preserving sharp edges and corners. They are able to preserve smooth regions of the signal avoiding the staircase effect as well. In this paper, we will introduce a new model based on the mean curvature regularizer and will leave the study of the Gaussian regularizer for a future work. 


\section{A new model for demodulating phase maps}

In this section we introduce a model based on the mean curvature of the phase map surface. The rationale of this selection is based upon argumentation of the previous section: a good phase regularizer should preserve the height of the phase surface for all scales. If this condition is not satisfied, the phase map may be a misrepresentation of the true phase map and less useful for metrology applications. The regularizer should also preserve edges and corners.

Therefore, we propose the following model to estimate the phase map, background illumination and amplitude terms

$$
\begin{aligned}
\underset{a, b, \phi}{\operatorname{argmin}} P(a, b, \phi, g) \equiv & \left\{\int_{\Omega}(u-g)^{2} d \Omega+\lambda_{1} \int_{\Omega}|\nabla a| d \Omega\right. \\
& \left.+\lambda_{2} \int_{\Omega}|\nabla b| d \Omega+\lambda_{3} \int_{\Omega}\left|\nabla \cdot \frac{\nabla \phi}{|\nabla \phi|}\right|^{p} d \Omega\right\},
\end{aligned}
$$

where $p=1,2$. In this paper, we will focus on the case $p=1$. We will call this model the mean curvature $(\mathrm{MC})$ model since we are using the mean curvature of the phase surface to regularize the phase maps. This model estimates with good accuracy phase maps in the interval $[0, \pi]$. To unfold wrapped phase maps, any of the many reported methods in the literature may be used.

\section{Preliminary analysis of the model}

In (3.1) we find three different subproblems, one for each variable, with different characteristics. To begin with, both subproblems for $a$ and $b$ have a TV regularizer which is known to be convex [17] while the mean curvature regularizer for $\phi$ makes the subproblem for this variable non convex.

The error term defined as $E(a, b, \phi, g)=\int_{\Omega}(u-g)^{2} d \Omega$, which is common to all three sub-problems, has the following second order optimality conditions for $a$ and $b$ :

$$
\begin{aligned}
& \frac{d^{2} E}{d a^{2}}=2 \int_{\Omega} d \Omega>0, \\
& \frac{d^{2} E}{d b^{2}}=2 \int_{\Omega} \cos ^{2}(\psi+\phi) d \Omega>0 .
\end{aligned}
$$

From (3.2), it is clear that $E$ is positive definite for $a$, hence following standard arguments [1], it can be shown the existence of a unique minimizer in the BV-space for this variable. From (3.3), we note that strict inequality is possible due to the presence of the carrier component. Therefore, $E$ is also positive definite for $b$ and a unique minimizer also exists.

Finally, a complete study of the subproblem for the phase map is not trivial due to the high order regularizer and non convexity of both: error term and regularizer. We remark 
that even with the disadvantages described above, the mean curvature regularized model presented in (3.1) has the very useful property of being independent of the signal height as proven in [29]. This feature helps the model to avoid decimating the signal height, a critical requirement for phase recovery algorithms. Further, curvature regularization does not suffer from staircasing while being able to keeping edges and contours sharp.

\section{Euler-Lagrange equations}

To find the minimizers of (3.1) we solve their associated Euler-Lagrange equations. Their derivation is presented in the Appendix at the end of the manuscript. They are given by

$$
\begin{aligned}
& -\nabla \cdot \frac{\nabla a}{|\nabla a|}+\lambda_{1}(u-g)=0, \\
& -\nabla \cdot \frac{\nabla b}{|\nabla b|}+\lambda_{2}(u-g) \cos (\psi+\phi)=0, \\
& \nabla \cdot\left(\frac{\nabla \Phi^{\prime}(\kappa)}{|\nabla \phi|}-\frac{\nabla \phi \cdot \nabla \Phi^{\prime}(\kappa)}{|\nabla \phi|^{3}} \nabla \phi\right)+\lambda_{3}(u-g)(-b \sin (\psi+\phi))=0,
\end{aligned}
$$

where $\kappa=-\nabla \cdot \frac{\nabla \phi}{\nabla \phi \phi}$ is the mean curvature of the level sets of $\phi, \Phi=\kappa^{2}$ or $\Phi=|\kappa|$ and with boundary conditions

$$
\frac{\partial a}{\partial v}=0, \quad \frac{\partial b}{\partial v}=0, \quad \frac{\partial \phi}{\partial v}=0,
$$

with $v$ the unit outward normal.

All of the above are highly nonlinear anisotropic partial differential equations (PDE). We note that the first two equations are second order PDEs while the last is fourth order.

Note that in order to avoid division by zero, we have to apply regularization by replacing $|\nabla a|$ in (3.4) with $|\nabla a|_{\beta}=\sqrt{|\nabla a|^{2}+\beta}$ where $\beta>0$ is a small parameter. A similar process is carried out in (3.5) and (3.6) to get $|\nabla b|_{\beta}$ and $|\nabla \phi|_{\beta}$.

\section{Numerical solution}

The numerical solution of the above three PDEs is very challenging due to their own nature. The well known and easy to implement Euler explicit algorithm has the drawback that the time-step needs to be selected very small for stability reasons. In particular, applying the Euler algorithm to solve (3.6) may take thousands of iterations, as reported in [3] for solving a similar problem, to reach a meaningful solution due to the stiffness of the PDE. Thus, the need of state of the art numerical algorithms. In this work we decided to use fixed point and Nesterov algorithms. Other techniques such as augmented Lagrangian methods [7, 14], convexity splitting [2], non linear multigrid [3], homotopy methods [28] and so on may result in a more efficient solution and will be part of our future work. 
We now proceed to describe in detail the fixed point algorithm for each one of the variables.

\section{A fixed point algorithm for $a$}

To solve (3.4), we adapted the fixed point method [26] to this new PDE. This is done by expressing (3.4) as a linear system of the form

$$
L_{a}\left(a^{k}\right) a^{k+1}=f_{a}
$$

for $k=0,1,2, \cdots$ as follows

$$
\left(-\nabla \cdot \frac{\nabla}{\left|\nabla a^{k}\right|}+\lambda_{1} I\right) a^{k+1}=\lambda_{1}(-b \cos (\psi+\phi)+g),
$$

where

$$
\begin{aligned}
& f_{a} \equiv \lambda_{1}(-b \cos (\psi+\phi)+g), \\
& L_{a}\left(a^{k}\right) \equiv-\nabla \cdot \frac{\nabla}{\left|\nabla a^{k}\right|}+\lambda_{1} I .
\end{aligned}
$$

An initial estimation $a^{0}$ is provided and a converging sequence of solutions $\left\{a^{k}\right\}_{k \geq 1}$ is constructed using (4.2). The nonlinear differential operator $L_{a}\left(a^{k}\right)$ is linearized by lagging the nonlinear coefficients $\left|\nabla a^{k}\right|^{-1}$ at every $k$-iteration. $L_{a}\left(a^{k}\right)$ is then positive definite and diagonally dominant. To solve the linear system at each iteration, we use Cholesky preconditioning together with the conjugate gradient (CG) method.

\section{A fixed point algorithm for $b$}

To solve (3.5), we apply the same approach by rewriting it as the following iterative equation:

$$
\left(-\nabla \cdot \frac{\nabla}{\left|\nabla b^{k}\right|}+\lambda_{2} \cos ^{2}(\psi+\phi)\right) b^{k+1}=\lambda_{2}(-a+g) \cos (\psi+\phi) .
$$

This time the right-hand side and the linear operator are defined by

$$
\begin{aligned}
& f_{b} \equiv \lambda_{2}(-a+g) \cos (\psi+\phi), \\
& L_{b}\left(b^{k}\right) \equiv-\nabla \cdot \frac{\nabla}{\left|\nabla b^{k}\right|}+\lambda_{2} \cos ^{2}(\psi+\phi) .
\end{aligned}
$$

In this case, $L_{b}\left(b^{k}\right)$ also has the nice property of being positive definite and diagonally dominant. The linear system

$$
L_{b}\left(b^{k}\right) b^{k+1}=f_{b}
$$

can also be solved with a preconditioned CG algorithm. 


\section{Nesterov accelerated gradient descent for $\phi$}

In our experiments, the fixed point method for $\phi$ did not work well, mainly due to the matrix not being positive definite nor diagonally dominant. Therefore, we decided to use Nesterov algorithm [15].

By defining the left hand side of (3.6) as $\nabla P(\phi)$, Nesterov accelerated gradient descent consists of the sequences in Algorithm 1.

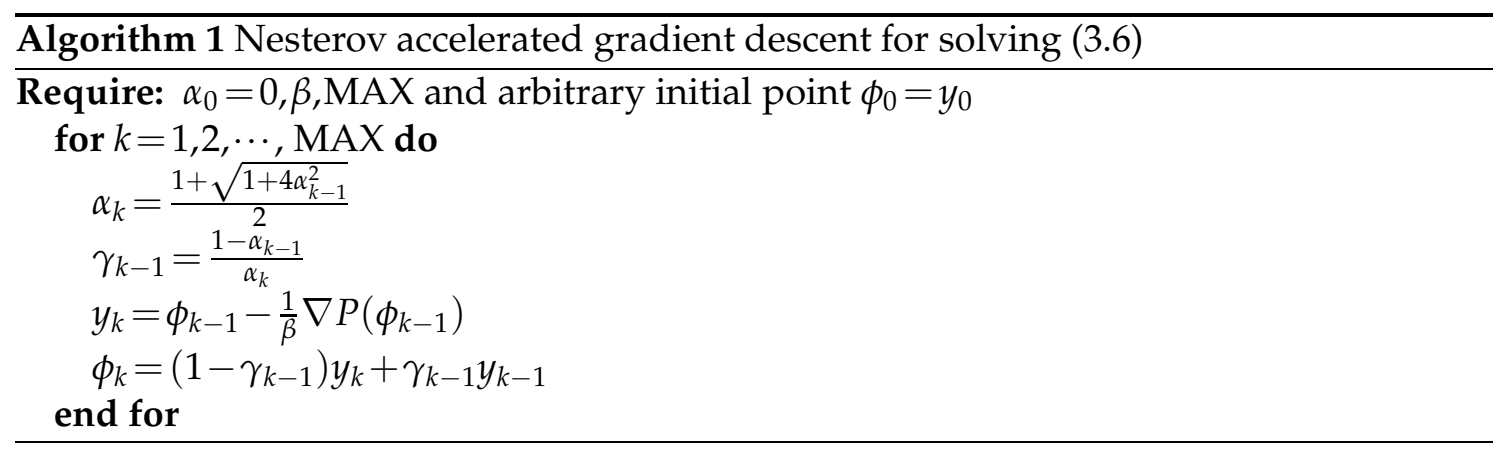

\section{The numerical algorithm}

Finally, to compute the minimizers of (3.1) we solve the three PDEs iteratively using a Gauss-Seidel process alike. This is made clear in Algorithm 2.

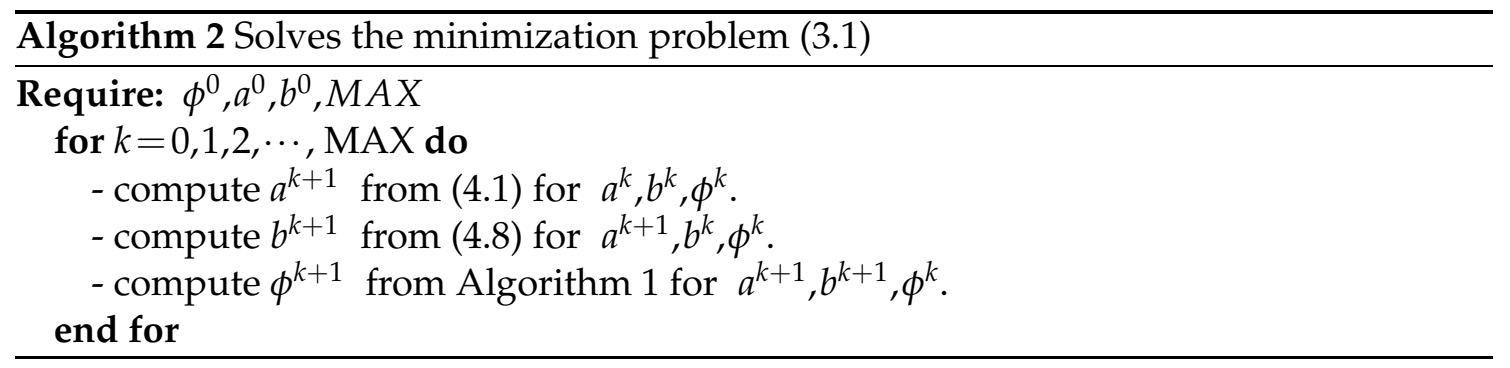

We note that rigorous mathematical proof of the convergence of Algorithm 2 and each one of the fixed point methods (4.1) and (4.8) is not ready and will be part of our future work. However, experimental evidence shows that this may be the case.

\section{Discretization}

We proceed to outline the discretization scheme we use. We choose the continuous domain as $\Omega=[0, m] \times[0, n]$ which ensures that the spatial steps are $h_{x}=h_{y}=1$ and let $\left(h_{x}, h_{y}\right)$ represent a vector of finite mesh sizes. Then we define the infinite grid by $G_{h}=\left\{(x, y): x=x_{i}=i h_{x}, y=y_{j}=j h_{y} ; i, j \in \mathbb{Z}\right\}$ and the cell-centered grid $\Omega_{h}=\Omega \cap G_{h}$. 
For illustration purposes we select $u$ to represent any continuous variable defined on $\Omega$. Then, its discrete version is given by $u_{h}=u_{h}(x, y)=u_{h}\left(x_{i}, y_{j}\right)=u_{h}\left(i h_{x}, j h_{y}\right)$. We will use $(\cdot)_{\varrho}$ to denote the derivative with respect to any variable $\varrho$.

To approximate the divergence operator, at some discrete point or pixel $(i, j)$, acting on any vector field $V=\left(V^{1}, V^{2}\right)$, i.e. $\nabla \cdot V=\left(V^{1}\right)_{x}+\left(V^{2}\right)_{y}$, we use central differences between ghost half-points as follows

$$
\nabla \cdot V_{i, j}=\frac{\left(V_{i+\frac{1}{2}, j}^{1}-V_{i-\frac{1}{2}, j}^{1}\right)}{h_{x}}+\frac{\left(V_{i, j+\frac{1}{2}}^{2}-V_{i, j-\frac{1}{2}}^{2}\right)}{h_{y}} .
$$

The mean curvature operator is approximated using

$$
\kappa_{i, j}=\frac{\left(u_{x}\right)_{i+\frac{1}{2}, j}}{|\nabla u|_{i+\frac{1}{2}, j}}-\frac{\left(u_{x}\right)_{i-\frac{1}{2}, j}}{|\nabla u|_{i-\frac{1}{2}, j}}+\frac{\left(u_{y}\right)_{i, j+\frac{1}{2}}}{|\nabla u|_{i, j+\frac{1}{2}}}-\frac{\left(u_{y}\right)_{i, j-\frac{1}{2}}}{|\nabla u|_{i, j-\frac{1}{2}}},
$$

partial derivatives in $x$ by the central differencing of two adjacent whole points

$$
\begin{aligned}
& \left(u_{x}\right)_{i+\frac{1}{2}, j}=\left(u_{i+1, j}-u_{i, j}\right) / h, \\
& \left(u_{x}\right)_{i-\frac{1}{2}, j}=\left(u_{i, j}-u_{i-1, j}\right) / h, \\
& \left(\Phi_{x}^{\prime}\right)_{i+\frac{1}{2}, j}=\left(\Phi_{i+1, j}^{\prime}-\Phi_{i, j}^{\prime}\right) / h, \\
& \left(\Phi_{x}^{\prime}\right)_{i-\frac{1}{2}, j}=\left(\Phi_{i, j}^{\prime}-\Phi_{i-1, j}^{\prime}\right) / h, \\
& |\nabla u|_{i+\frac{1}{2}, j}=\sqrt{\left(\left(u_{x}\right)_{i+\frac{1}{2}, j}\right)^{2}+\left(\left(u_{y}\right)_{i, j+\frac{1}{2}}\right)^{2}+\beta},
\end{aligned}
$$

partial derivatives in $y$ by the min-mod of $(\cdot)_{y}$ 's at two adjacent whole points

$$
\begin{aligned}
& \left(u_{y}\right)_{i+\frac{1}{2}, j}=\min -\bmod \left(\frac{1}{2 h}\left(u_{i+1, j+1}-u_{i+1, j-1}\right), \frac{1}{2 h}\left(u_{i, j+1}-u_{i, j-1}\right)\right) \\
& \left(u_{y}\right)_{i-\frac{1}{2}, j}=\min -\bmod \left(\frac{1}{2 h}\left(u_{i, j+1}-u_{i, j-1}\right), \frac{1}{2 h}\left(u_{i-1, j+1}-u_{i-1, j-1}\right)\right) \\
& \left(\Phi_{y}^{\prime}\right)_{i+\frac{1}{2}, j}=\min -\bmod (\zeta, \vartheta) \text { with } \\
& \zeta=\frac{1}{2 h}\left(\Phi_{i+1, j+1}^{\prime}-\Phi_{i+1, j-1}^{\prime}\right) \text { and } \vartheta=\frac{1}{2 h}\left(\Phi_{i, j+1}^{\prime}-\Phi_{i, j-1}^{\prime}\right) \\
& \left(\Phi_{y}^{\prime}\right)_{i-\frac{1}{2}, j}=\min -\bmod (\zeta, \vartheta) \text { with } \\
& \zeta=\frac{1}{2}\left(\Phi_{i, j+1}^{\prime}-\Phi_{i, j-1}^{\prime}\right) \text { and } \vartheta=\frac{1}{2}\left(\Phi_{i-1, j+1}^{\prime}-\Phi_{i-1, j-1}^{\prime}\right) \\
& |\nabla u|_{i-\frac{1}{2}, j}=\sqrt{\left(\left(u_{x}\right)_{i-\frac{1}{2}, j}\right)^{2}+\left(\left(u_{y}\right)_{i, j-\frac{1}{2}}\right)^{2}+\beta}
\end{aligned}
$$


By using a similar procedure, we can obtain the approximations for $V_{i, j+\frac{1}{2}}^{2}$ and $V_{i, j-\frac{1}{2}}^{2}$. Finally, the Neumann's boundary condition on $\partial \Omega$ is treated as

$$
u_{i, 0}=u_{i, 1}, \quad u_{i, n+1}=u_{i, n}, \quad u_{0, j}=u_{1, j}, \quad u_{m+1, j}=u_{m, j} .
$$

The min-mod function is defined below

$$
\min -\bmod (a, b)=\left(\frac{\operatorname{sgn} a+\operatorname{sgn} b}{2}\right) \min (|a|,|b|) \text {. }
$$

\section{Experimental results}

In this section we present some experimental results to illustrate the virtues of our model.

We begin with the synthetic problem shown in Fig. 1(a), consisting of a vertical fringe pattern as the ones used in metrology in the semiconductor industry. The results of applying to this problem the TV model (2.1) and the MC model (3.1) with different values of the regularization parameter $\lambda_{3}$ are shown in the left and right columns of Fig. 2, respectively. It can be seen that for the right choice of the parameter, i.e. $\lambda_{3}=0.001$ both models deliver a very good reconstruction of the phase map. However, when the parameter value is increased, the TV model starts suffering from loss of signal height while the MC model manages to deliver a much better result. This is important since the right value of $\lambda_{3}$ is not known in advance and usually difficult to find.

Now we move to illustrate the good performance of our MC model to changes of feature scale. In Fig. 1(b), we show a synthetic problem including a phase map with features at two different scales. On one hand, the results of applying model (2.1) to recover the phase map are shown in the first two rows (from top to bottom) of Fig. 3. In the first row,

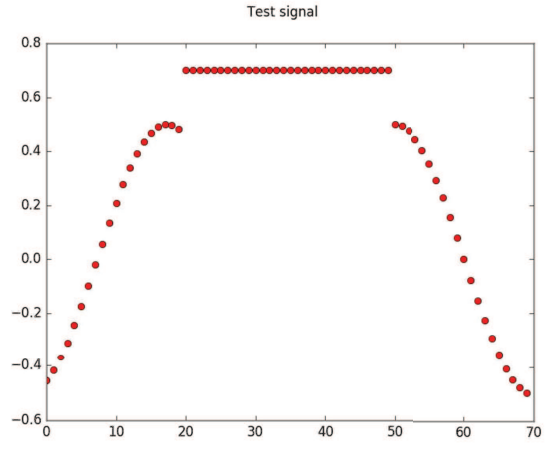

(a) Test problem 1

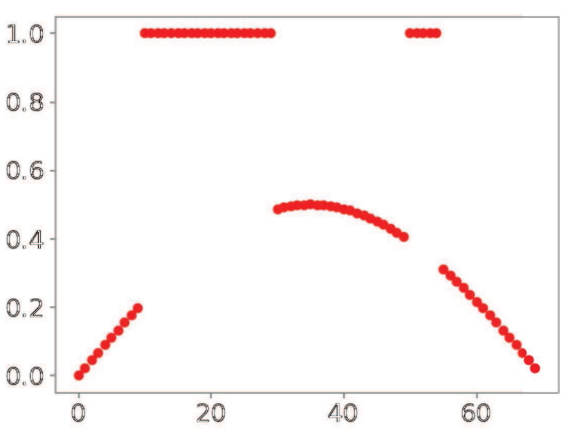

(b) Test problem 2

Figure 1: Test problem 1 is a synthetic piecewise signal with constant and smooth regions. Test problem 2 is a synthetic piecewise signal with features at two different scales. 

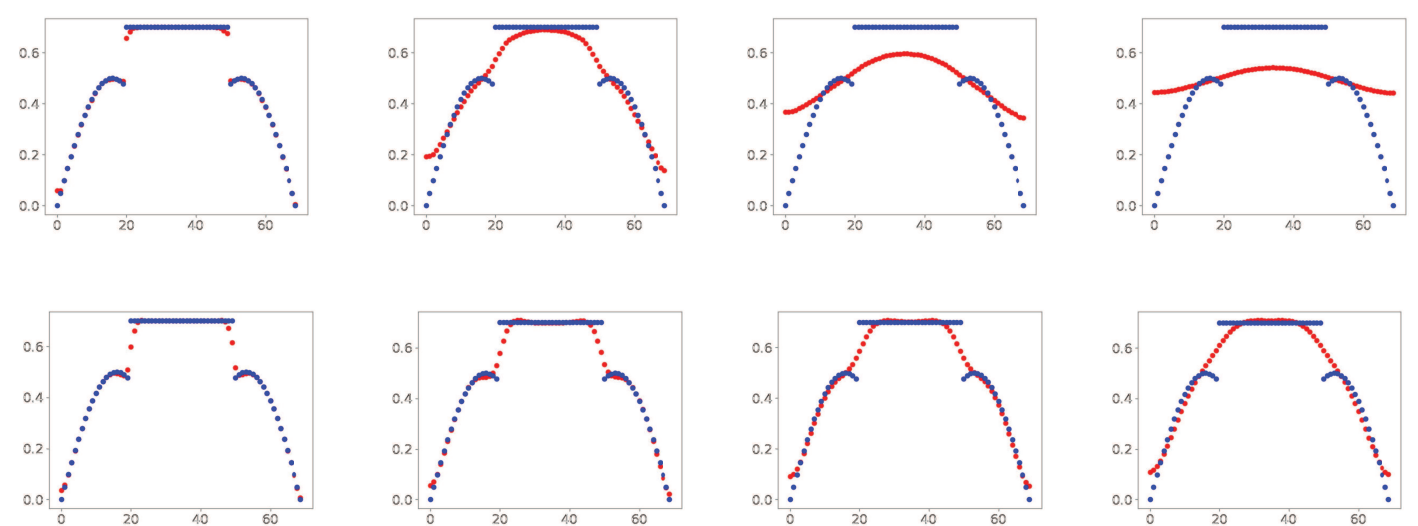

Figure 2: In all plots, the red dotted line is the recovered phase map and the blue dotted line the ground truth. The results from the TV model (2.1) are presented in the top row and from the MC model (3.1) in the bottom row. From left to right, the values for the regularization parameter were selected as follows: first column $\lambda_{3}=0.01$, second column $\lambda_{3}=1$, third column $\lambda_{3}=10$, fourth column $\lambda_{3}=100$.
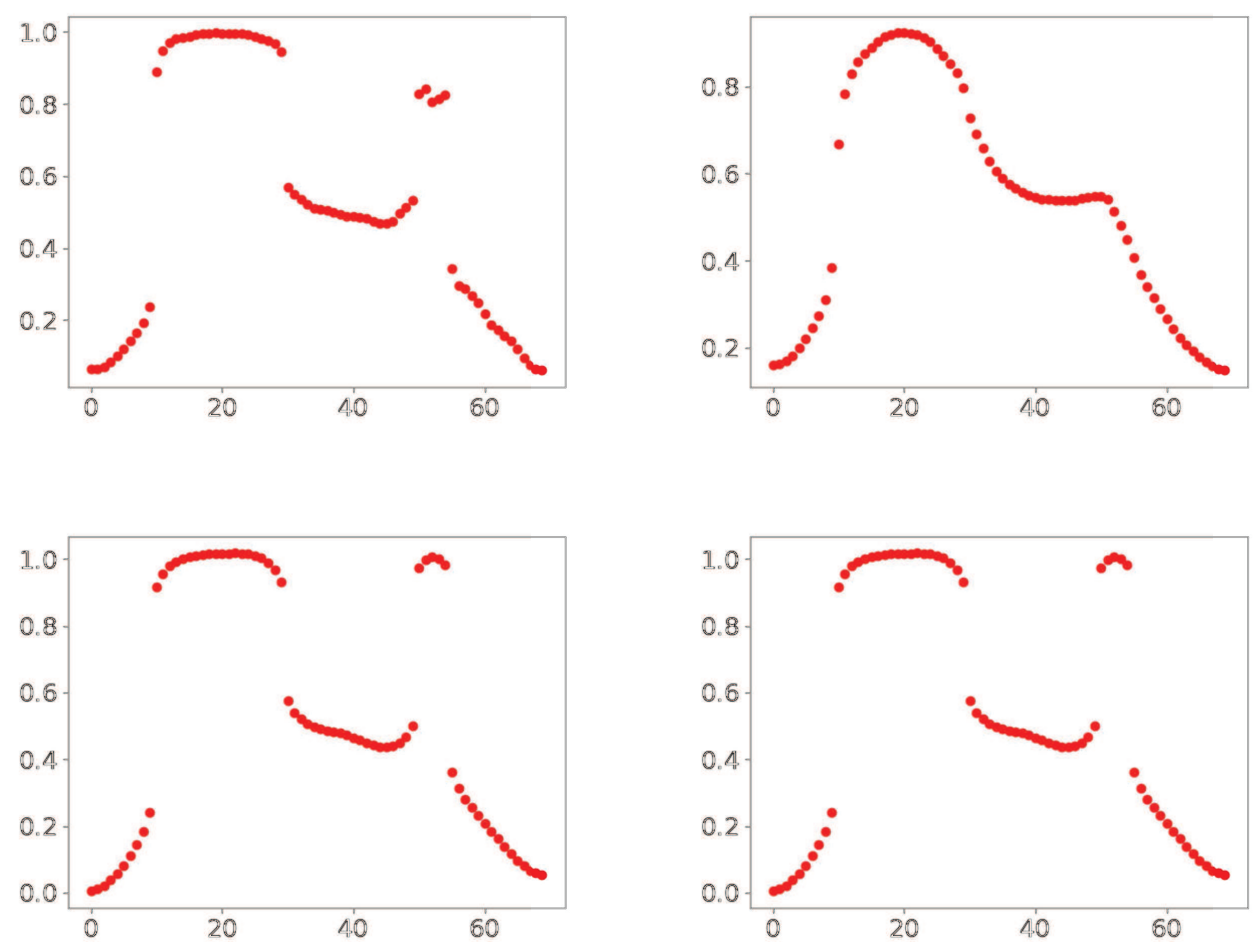

Figure 3: Top row, are the results for the TV regularized model using $\lambda_{3}=0.05$ and $\lambda_{3}=0.25$ respectively. The bottom row are the results for the MC model using the same values of $\lambda_{3}$. 

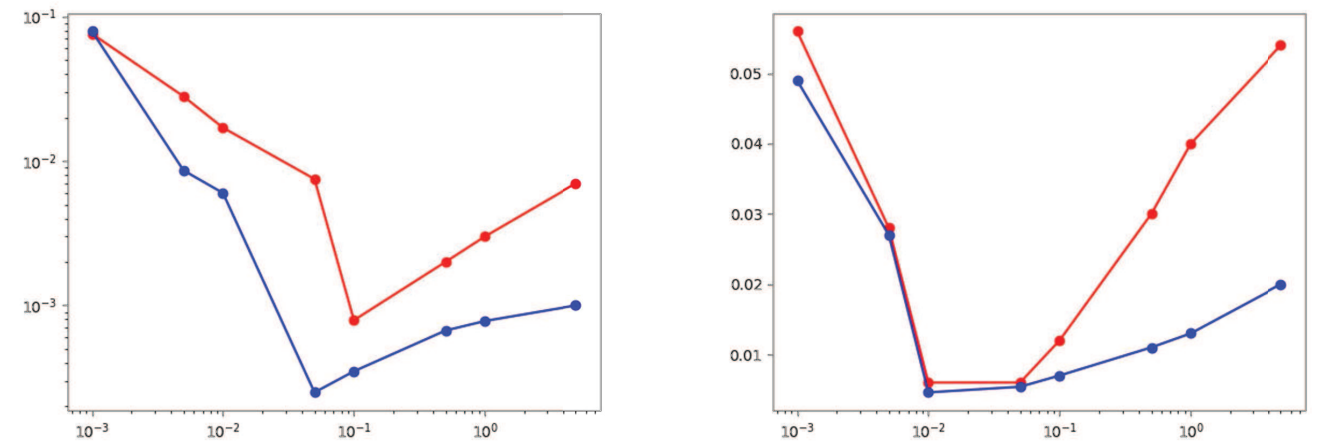

Figure 4: To the left the MSE between the ground truth phase and the recovered phase from the TV model for Test problem 1. To the right, the same for the Test problem 2. In both plots, the red line is the MSE for the TV base model while the blue line is the MSE for the MC model. Clearly, the MC model, outperforms in both cases the TV model by having a smaller MSE. The values of $\lambda_{3}$ tested are in the interval $[0.001,5]$.

where $\lambda_{3}=0.05$ has been used, it can be seen that the TV model is incapable of recovering the right height of the feature with the smallest scale and some staircasing is present. The second row shows how the TV model completely wipes out the feature with smallest scale when the regularized parameter is increased to $\lambda_{3}=0.25$. On the other hand, the results of using our MC model (3.1) are shown in the third and fourth rows. The same values for the regularization parameter $\lambda_{3}=0.05$ and $\lambda_{3}=0.25$ were used. In contrast to the TV model, the MC model delivered a very good phase recovering without decimating the signal heights no matter the scale of the features.

A good selection of the regularization parameter in a variational model maybe critical to obtaining good results. We suggest to use the L-curve method [27] to select all regularization parameters in the MC based model. To illustrate the performance of the TV and MC models for different values of $\lambda_{3}$, we present some results in Fig. 4 where we plot the mean square error (MSE) for both models. It can be seen that for almost all values of $\lambda_{3}$, the MC model performs better than the TV model and is less affected by overregularization.

We also present an experiment consisting of processing a fringe pattern obtained from a holographic interferometry experiment [11], which consisted of the height measurement of a micro-thin film. The fringe pattern obtained from this experiment is shown in Fig. 5(a). The fringe pattern was demodulated using Fourier-based quadrature transform method described in [21]. During the demodulation process, first the following terms are estimated: background, modulation, and spatial carrier. Then, we subtract the carrier term from the wrapped phase and the phase term is unwrapped if needed [11]. These estimations are used as initial values in our method. On one hand, comparing the result of our model, shown in Fig. 5(d), against the result obtained from the very well known Schwinder-Hariharan algorithm [11] and shown in Fig. 5(b), we first remark that our 


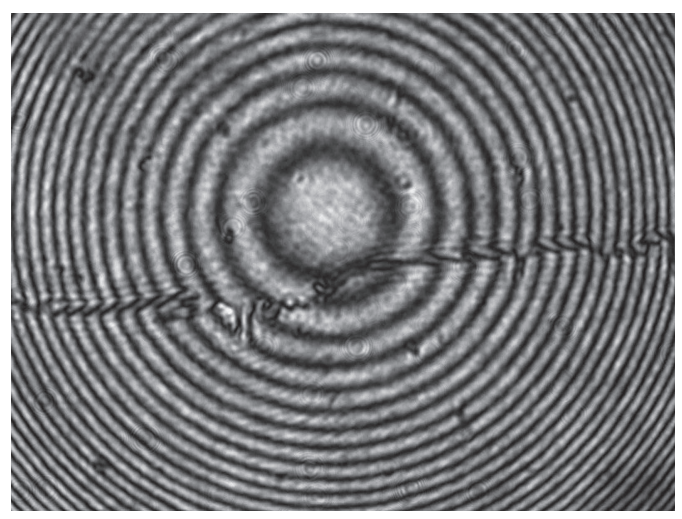

(a) Experimental fringe pattern

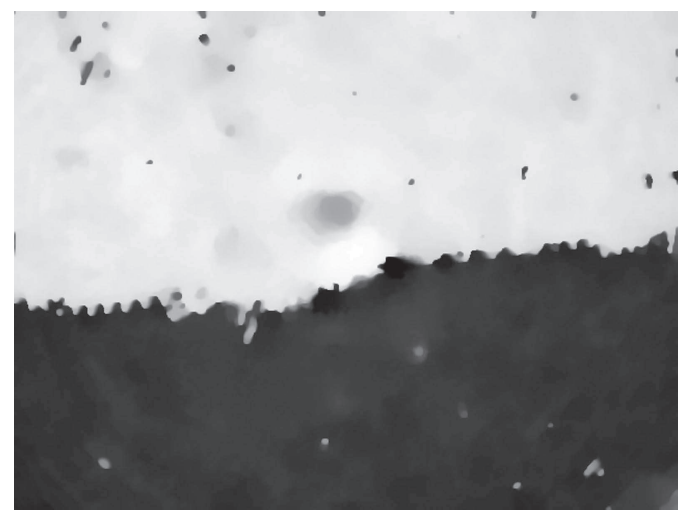

(c) Estimated phase using the TV regularized model [17]

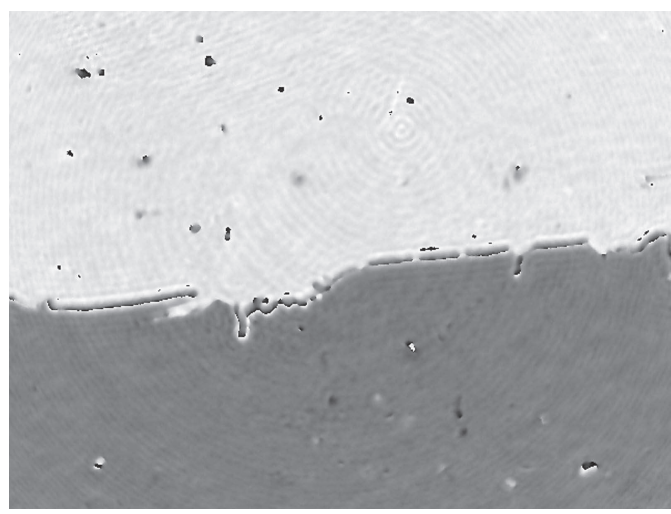

(b) Estimated phase using the SchwinderHariharan algorithm

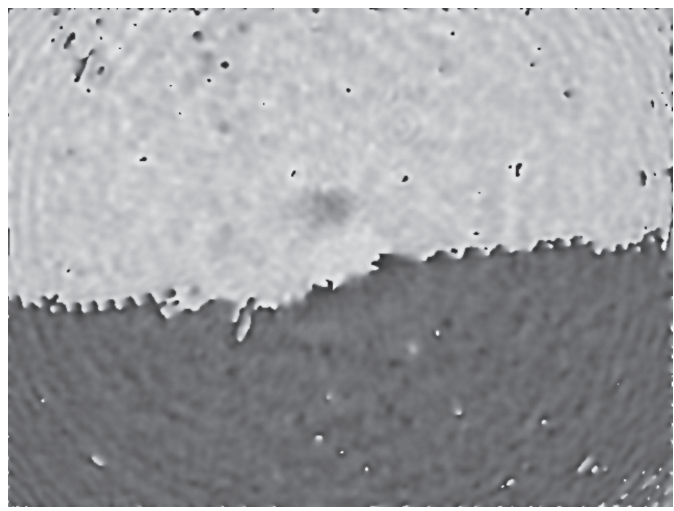

(d) Estimated phase using our curvature based model

Figure 5: (a) An experiment consisting of processing a fringe pattern obtained from a holographic interferometry experiment, which consisted of the height measurement of a micro-thin film. In (b), (c) and (d) are the resulting phase maps from the Schwinder-Hariharan algorithm, the TV model and the MC model respectively.

model is able to deliver a similar phase recovery by preserving the dynamic range of the phase. On the other hand, comparing the result of our model against the result from the TV regularized model, shown in Fig. 5(c), we appreciate that they differ in smoothness, i.e. model (2.1) delivers a piecewise constant signal while model (3.1) a smoother signal. An important remark here is that our model is equipped with the right tool to recover small scale features, something that the Schwinder-Hariharan and TV algorithms most likely will miss.

Finally, we warn that our model has multiple solutions due to the non convexity of the mean curvature regularizer. This may pose a problem since variations in $\phi$ as well as $b$ may explain the interference pattern. To solve this problem, a numerical algorithm capable of finding the global minimum is the solution. In [23], the authors reported that 
multigrid method is more likely to converge to the global minimizer of a particular nonconvex variational problem than unilevel methods. Therefore, in the future we will be implementing a multigrid algorithm for the MC model.

\section{Conclusions}

We presented a new variational model for estimating phase maps from fringe patterns. The new model differs from others in that it uses a mean curvature regularizer for the phase map. Up to our knowledge this is the very first work to introduce a curvature regularizer for phase estimation. Three Euler-Lagrange equations to minimize the model were derived and fast numerical algorithms for their solution presented. Experimental results over two synthetic problems and one real life problem were presented to illustrate the main virtue of our model: signal height or dynamic range preservation to different feature scales. Part of our future work involves developing even faster algorithms such as nonlinear multigrids and augmented Lagrangian methods.

\section{Appendix}

Here, we derive the first-order optimality conditions or Euler-Lagrange equations for $a, b$ and $\phi$. In the formal derivation we assume that the vector fields are smooth enough such that gradients are well defined and the variation $\varphi$ has compact support over $\Omega$ so that we can use the divergence theorem to get rid of the boundary terms.

\section{Euler-Lagrange equation for $\phi$}

We start with the regularizer $R(\phi)=\int_{\Omega} \Phi(\kappa)$ given in (3.1) and compute the first variation as it is customary using $\left.\delta R_{\phi} \equiv \frac{d}{d \epsilon} R(\phi+\epsilon \varphi)\right|_{\epsilon=0}$. For convenience, we write $\langle f\rangle=\int_{\Omega} f d \Omega$ and the boundary integral as $\langle f\rangle_{\partial}=\int_{\partial \Omega} f d s$ with $d s$ denoting the arc-length element. The the variation of $R$ is

$$
\begin{aligned}
\delta R_{\phi} & =\langle\delta(\Phi(\kappa))\rangle \\
& =\left\langle\Phi^{\prime}(\kappa) \delta(\kappa)\right\rangle \\
& =\left\langle\Phi^{\prime}(\kappa) \delta\left(\nabla \phi \cdot \frac{\nabla \phi}{|\nabla \phi|}\right)\right\rangle \\
& =\left\langle\Phi^{\prime}(\kappa) \nabla \cdot\left[\frac{1}{|\nabla \phi|} \nabla(\delta \phi)+\nabla \phi \delta\left(\frac{1}{|\nabla \phi|}\right)\right]\right\rangle \\
& =\left\langle\Phi^{\prime}(\kappa) \nabla \cdot\left[\frac{1}{|\nabla \phi|} \nabla(\delta \phi)-\frac{\nabla \phi}{|\nabla \phi|^{3}}(\nabla \phi \cdot \nabla(\delta \phi))\right]\right\rangle .
\end{aligned}
$$


We note that $\frac{\nabla(\delta \phi) \cdot \nabla \phi}{|\nabla \phi|} \frac{\nabla \phi}{|\nabla \phi|}$ is the projection of $\nabla \delta \phi$ onto $\nabla \phi$ and therefore we can write $\delta R$ as follows

$$
\delta R_{\phi}=\left\langle\Phi^{\prime}(\kappa) \nabla \cdot\left[\frac{1}{|\nabla \phi|} \nabla(\delta \phi)-\frac{1}{|\nabla \phi|} \Pi \nabla(\delta \phi)\right]\right\rangle
$$

where $\Pi$ is the projection operator onto the normal direction. Then by using the divergence theorem we get

$$
\delta R_{\phi}=\left\langle-\frac{\nabla \Phi^{\prime}(\kappa)}{|\nabla \phi|} \nabla(\delta \phi)+\Pi \nabla \Phi^{\prime}(\kappa) \frac{1}{|\nabla \phi|} \nabla(\delta \phi)\right\rangle,
$$

where we have dropped the boundary integrals

$$
\left\langle\Phi^{\prime}(\kappa) \frac{\nabla(\delta \phi)}{|\nabla \phi|} \cdot v\right\rangle_{\partial} \text { and }\left\langle\Phi^{\prime}(\kappa) \Pi \frac{\nabla(\delta \phi)}{|\nabla \phi|} \cdot v\right\rangle_{\partial}^{\prime}
$$

and $v$ is the unit outward normal vector. Now we apply the divergence theorem again getting

$$
\delta R_{\phi}=\left\langle\nabla \cdot \frac{\nabla \Phi^{\prime}(\kappa)}{|\nabla \phi|} \delta \phi-\nabla \cdot \frac{\Pi \nabla \Phi^{\prime}(\kappa)}{|\nabla \phi|} \delta \phi\right\rangle,
$$

where this time we dropped the following boundary integrals

$$
\left\langle\frac{\nabla \Phi^{\prime}(\kappa)}{|\nabla \phi|} \delta \phi \cdot v\right\rangle_{\partial} \text { and }\left\langle\frac{\Pi \nabla \Phi^{\prime}(\kappa)}{|\nabla \phi|} \delta \phi \cdot v\right\rangle_{\partial} .
$$

From (A.5), we get the first order optimality condition

$$
\nabla \cdot\left(\frac{\nabla \Phi^{\prime}(\kappa)}{|\nabla \phi|}-\frac{\Pi \nabla \Phi^{\prime}(\kappa)}{|\nabla \phi|}\right)=0
$$

By applying $\Pi$ to $\nabla \Phi^{\prime}(\kappa)$ we get

$$
\nabla \cdot\left(\frac{\nabla \Phi^{\prime}(\kappa)}{|\nabla \phi|}-\frac{\nabla \Phi^{\prime}(\kappa) \cdot \nabla \phi}{|\nabla \phi|^{3}} \nabla \phi\right)=0
$$

just like in (3.6).

We now turn to compute the first variation $\delta F_{\phi}$ of the error term $F=\int_{\Omega}(u-g)^{2} d \Omega$ with respect to $\phi$

$$
\begin{aligned}
\delta F_{\phi} & =\left\langle\delta\left((a+b \cos (\psi+\phi)-g)^{2}\right)\right\rangle \\
& =\langle 2((a+b \cos (\psi+\phi)-g)(-b \sin (\psi+\phi)) \delta \phi\rangle,
\end{aligned}
$$

obtaining the following optimality condition

$$
2(a+b \cos (\psi+\phi)-g)(-b \sin (\psi+\phi)=0 .
$$

From (A.8) and (A.10) we obtain the Euler-Lagrange equation for $\phi$ given in (3.6). 


\section{Euler-Lagrange equations for $a, b$}

For completeness we show how to compute the Euler-Lagrange equations for $a$ and $b$. Similar as before we compute the variations $\delta F_{a}, \delta F_{b}$ with respect to $a$ and $b$ respectively

$$
\begin{aligned}
& \delta F_{a}=\langle 2((a+b \cos (\psi+\phi)-g) \delta a\rangle, \\
& \delta F_{b}=\langle 2((a+b \cos (\psi+\phi)-g) \cos (\psi+\phi) \delta b\rangle .
\end{aligned}
$$

The variation for the regularizers $R_{a}=\int_{\Omega}|\nabla a| d \Omega$ and $R_{b}=\int_{\Omega}|\nabla b| d \Omega$ it is pretty standard and can be found in many papers see for instance [20]. It is given by

$$
\begin{aligned}
\delta R_{a} & =\langle\delta(|\nabla a|)\rangle \\
& =\left\langle\frac{\nabla a}{|\nabla a|} \delta(\nabla a)\right\rangle \\
& =\left\langle-\nabla \cdot \frac{\nabla a}{|\nabla a|} \delta a\right\rangle,
\end{aligned}
$$

where we have dropped the boundary integral

$$
\left\langle\frac{\nabla a}{|\nabla a|} \delta a \cdot v d s\right\rangle_{\partial}
$$

Therefore the optimality condition is given by

$$
-\nabla \cdot \frac{\nabla a}{|\nabla a|}=0
$$

and similar for $b$.

Putting all together, (A.12), (A.11) and (A.15) we get the Euler-Lagrange equations (3.4) and (3.5).

\section{References}

[1] Acar, R., and Vogel, C. R. Analysis of bounded variation penalty methods for ill-posed problems. Inverse problems., 10(6), 1217, (1994).

[2] Jessica Bosch, David Kay, Martin Stoll, and Andrew J. Wathen. Fast Solvers for CahnHilliard Inpainting. SIAM J. Imaging Sciences, 7(1), 67-97, (2014).

[3] Carlos Brito-Loeza and Ke Chen. Multigrid Algorithm for High Order Denoising. SIAM Journal on Imaging Sciences, Volume 3 (3), pp.363-389, (2010).

[4] C. Brito-Loeza, K. Chen, V. Uc-Cetina. Image denoising using the Gaussian curvature of the image surface. Numerical Methods for Partial Differential Equations, 32(3), 1066-1089, (2015).

[5] Chan, T., Esedoglu, S., Park, F., and Yip, A. Recent developments in total variation image restoration. Handbook of Mathematical Models of Computer Vision., 17-31, (2005).

[6] Tony Chan, Antonio Marquina, and Pep Mulet. High-Order Total Variation-Based Image Restoration. SIAM Journal on Scientific Computing, 22(2), 503-516, (2000). 
[7] Chunlin Wu, Juyong Zhang, Yuping Duan, Xue-Cheng Tai. Augmented Lagrangian Method for Total Variation Based Image Restoration and Segmentation Over Triangulated Surfaces. SIAM Journal on Scientific Computing, 50(1), 145-166, (2012).

[8] D. J. Eyre. Unconditionally gradient stable time marching the Cahn-Hilliard equation. Computational and Mathematical Models of Microstructural Evolution, edited by J. W. Bullard et al. Materials Research Society, Warrendale, PA, pp. 39-46, 1998.

[9] C. Galvan and M. Rivera. Second-order robust regularization cost function for detecting and reconstructing phase discontinuities. Appl. Opt., 45, 353359 (2006).

[10] vladimir Katkovnik, Igor Shevkunov, Nikolay V. Petrov, and Karen Egiazarian. Highaccuracy off-axis wavefront reconstruction from noisy data: local least square with multiple adaptive windows. Opt. Express., 4, 25068-25083 (2016).

[11] T. Kreis. Holographic Interferometry: Principles and Methods. Wiley-VCH., (1996).

[12] A. Marquina and S. Osher. Explicit algorithms for a new time dependent model based on level set motion for nonlinear deblurring and noise removal. SIAM J. Sci. Comput., 22(2):387$405,2000$.

[13] J. L. Marroquin, M. Rivera, S. Botello, R. Rodriguez-Vera, and M. Servin. Regularization methods for processing fringe-pattern images Appl. Opt., 38, 788794 (1999).

[14] M. Myllykoski, R. Glowinski, T. Kärkkäinen and T. Rossi. A New Augmented Lagrangian Approach for L1-mean Curvature Image Denoising. SIAM J. Imaging Sciences, 8(1), 95-125, (2015).

[15] Yu. Nesterov. Introductory Lectures on Convex Optimization. A Basic Course. Springer, ISBN 1-4020-7553-7 (2004).

[16] M. Nikolova. Local strong homogeneity of a regularized estimator. SIAM J. Appl. Math., 61, 633-658, (2000).

[17] R. Legarda-Saenz, C. Brito-Loeza, A. Espinosa-Romero. Total variation regularization cost function for demodulating phase discontinuities. Applied Optics, 53(11), 2297-2301, (2014).

[18] R. Legarda-Saenz, W. Osten, and W. P. Juptner. Improvement of the regularized phase tracking technique for the processing of non-normalized fringe patterns. Appl. Opt., 41, 55195526 (2002).

[19] R Oven. Measurement of planar refractive index profiles with rapid variations in glass using interferometry and total variation regularized differentiation. Journal of Modern Optics, 62:sup2, S53-S60, (2015).

[20] L. I. Rudin, S. Osher, and E. Fatemi. Nonlinear total variation based noise removal algorithms. Physica D, 60:259-268, (1992).

[21] M. Servin, J. A. Quiroga, and J. L. Marroquin General n-dimensional quadrature transform and its application to interferogram demodulation. J. Opt. Soc. Am.., a 20, 925-934, (2003).

[22] Mandeep Singh and Kedar Khare Single-shot interferogram analysis for accurate reconstruction of step phase objects. J. Opt. Soc. Am. A, 34, 349-355 (2017).

[23] Noor Badshah and Ke Chen. Multigrid Method for the Chan-Vese Model in Variational Segmentation. Communications in Computational Physics, 4(2), 294-316, (2009).

[24] David Strong and Tony Chan. Edge-preserving and scale-dependent properties of total variation regularization. Inverse Problems, 19(6) S165-S187, (2003).

[25] J. Villa, J. A. Quiroga, and M. Servin Improved regularized phase-tracking technique for the processing of squared-grating deflectograms. Appl. Opt., 39, 502508 (2000).

[26] C.R. Vogel and M.E. Oman. Computational Methods for Inverse Problems. SIAM J. Sci. Comput., 17:227-238, (1996).

[27] C.R. Vogel Iterative algorithms for total variation denoising. Society for Industrial and Applied 
Math., 1st edition, Philadelphia, PA, USA, 2002.

[28] F L Yang, Ke Chen and Bo Yu. Homotopy method for a mean curvature-based denoising model. Journal of Applied Numerical Mathematics, Vol 62 (3), pp.185-200, (2012).

[29] Wei Zhu and Tony Chan. Image denoising using mean curvature of image surface. SIAM J. Imaging Sci., 5, 1-32, 2012.

[30] Zhu, X., Tang, C., Li, B., Sun, C., and Wang, L. Phase retrieval from single frame projection fringe pattern with variational image decomposition. Optics and Lasers in Engineering., 59, 25-33, (2014). 